\title{
A Survey on Descendants of LEACH Protocol
}

\author{
Prashant Maurya, Amanpreet Kaur \\ Central University of Punjab, Bathinda, 151001, India \\ E-mail: \{prashant.glaitm, pandheraman\}@gmail.com
}

\begin{abstract}
A wireless sensor network (WSN) is an emerging field comprising of sensor nodes as basic units. These sensor nodes have limited resources like power, memory etc. WSNs can be used to monitor the remote areas where recharging or replacing the battery power of sensor nods is not possible. This limitation of WSNs makes energy consumption as a most challenging issue. Low-Energy Adaptive Clustering Hierarchy (LEACH) is an easiest and first significant protocol which consumes less amount of energy while routing the data to the base station. A lot of work has been done to improve energy efficiency of routing protocol by taking LEACH as a base protocol. In this review paper section I has introduction to Wireless Sensor Networks, section II has introduction of LEACH Protocol and all descendant protocols of LEACH with comparison table have been discussed in section III.
\end{abstract}

Index Terms-LEACH, Energy Adaptive Protocol, WSN, Routing Protocol, Cluster-Head.

\section{INTRODUCTION}

Nowadays, wireless sensor network (WSN) is emerging as a promising and interesting area. A WSN consists of few sensor nodes which have sensing and computational capabilities. These sensor nodes are very efficient in terms of power usage and wireless communication and available at low cost. These sensor nodes can sense the environment conditions like temperature, humidity, pressure, sound, movement, pollutants and many other conditions like seismic, low sampling rate, thermal visual, infrared, acoustic and radar [1]. According to these different types, a WSN can be applied to monitor many military and civilian environments. In wireless sensor network a wireless medium is used by the nodes to communicate with each other. Each sensor node has a battery associated with it to provide power supply for sensor unit. Sensor nodes can be deployed in the sensing region to sense those environment where recharging and replacing of battery is not possible. These nodes work cooperatively to collect and forward the collected data to the sink. The sensor nodes are deployed in the sensing area through wireless links which provide opportunities for many civilian and military applications, for example: intrusion detection, battlefield monitoring and availability of equipment, environment observation and home intelligence.

Wireless sensor network can be categorized into two types based on node deployment strategy as unconstructed WSN and structured WSN. In unstructured WSN the sensor nodes are densely deployed and or they can be deployed in ad-hoc manner in the sensing area or region. While in structured WSN the sensor node deployment of some or all nodes are pre-planned. The nodes placement is also planned. So, the maintenance of structured WSN is much easy as compare to Unstructured WSN because deployed locations of nodes are known [2].

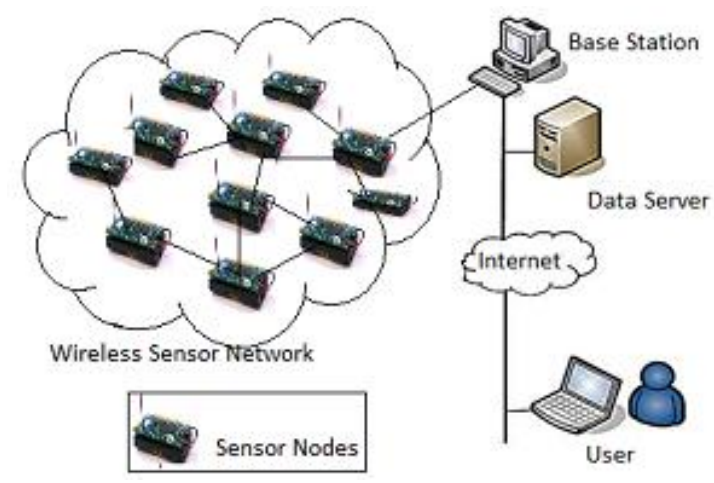

Fig.1. Wireless Sensor Network Scenario

\section{LEACH PROTOCOL}

Low-Energy Adaptive Clustering Hierarchy (LEACH) is most significant hierarchical and cluster based protocol in wireless sensor network. The main objective of LEACH is to minimize the energy consumption in sensor networks.

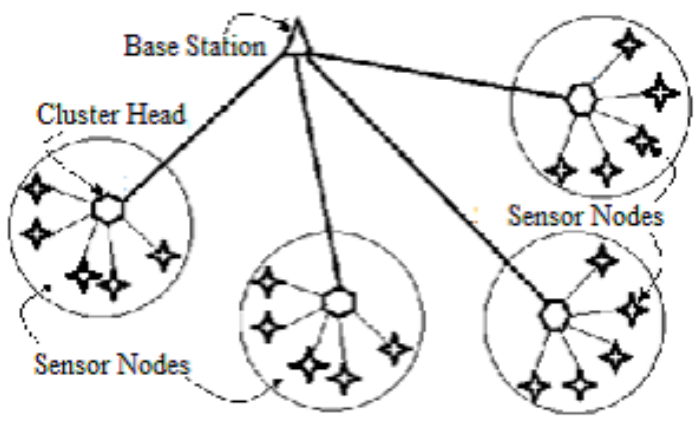

Fig.2. Architecture of LEACH Protocol

In LEACH Protocol nodes are randomly distributed in the field with capability of gathering and processing the data. LEACH protocol proceeds into several rounds which is further divided into two phases as setup phase and steady state phase. 


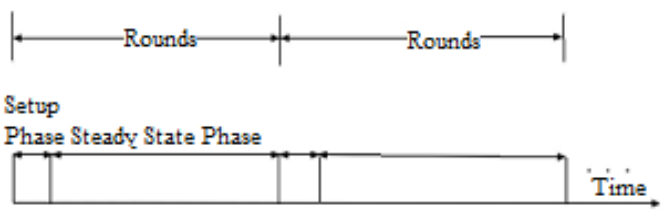

Fig.3. A round in LEACH Protocol

During the set-up phase, each sensor node chooses a random number between 0 and 1.If this random number is lower than the threshold for node $\mathrm{n}$, then node become a cluster head node $(\mathrm{CH})$. The threshold value $\mathrm{T}(\mathrm{n})$ of node can be calculated using following formula:

$$
T(n)=\frac{p}{1-p\left(r \bmod \left(\frac{1}{p}\right)\right)} \text { if } n \in G
$$

Where $T(n)$ is the threshold value of node $n$ and $G$ is set of nodes which are not have been $\mathrm{CH}$ in last $1 / \mathrm{p}$ rounds. When $\mathrm{CH}$ has selected successfully, it broadcasts an advertisement message to the other nodes. Based on the received signal strength of the advertisement, other nodes decide to which cluster it will join for this round and send a membership message to its $\mathrm{CH}$. Now cluster formation completed.

During the Steady State phase, normal nodes collects and sends data to its corresponding $\mathrm{CH}$. data transmission takes place based on the TDMA schedule and the clusterheads performs data aggregation through local computation.

LEACH uses following radio model in which the transmitting and receiving operations consume energy according to following formula.

$$
\begin{gathered}
E_{T X}(k, d)=E_{T X-e l e c}(k)+E_{T X-a m p}(k, d) \\
E_{T X-e l e c}(k)=E_{\text {elec }} * k \\
E_{T X-a m p}(k, d)=\left\{\begin{array}{l}
\varepsilon_{f S} * d^{2} * k \text { if } d<d_{0} \\
\varepsilon_{f S} * d^{4} * k \text { if } d \geq d_{0}
\end{array}\right. \\
E_{R X}(k)=E_{R X_{-} \text {elec }}(k)=E_{\text {elec }} * k \\
d_{0}=\sqrt{\frac{\varepsilon_{f s}}{\varepsilon_{\text {amp }}}}
\end{gathered}
$$

Where $k$ is the message size and $d$ is the distance.

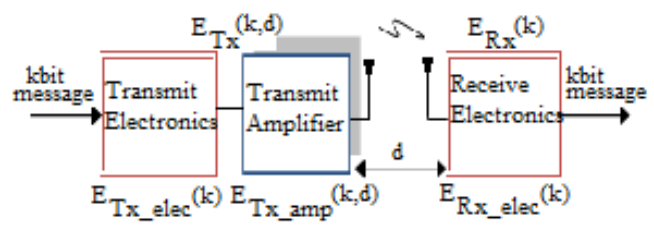

Fig.4. Energy Model

\section{A. Advantage of LEACH:}

- LEACH is a completely distributed approach.

- It does not require any global information of network.

- It is a powerful and simple routing protocol.

- It uses random rotation of cluster-Head, which provides each node to become a cluster head node in a round.

- It uses TDMA so that each node can participate in rounds simultaneously.

- Each sensor node communicates only with associated cluster head $(\mathrm{CH})$. It provides localized co-ordination and control for cluster setup and operation.

- Only a cluster head node $(\mathrm{CH})$ aggregates the data collected by the nodes to minimize the data redundancy.

\section{B. Disadvantage of LEACH:}

- In LEACH Protocol only cluster head $(\mathrm{CH})$ is responsible for sending data to base station (BS) directly. So, failure of $\mathrm{CHs}$ leads to lack of robustness.

- Single Hop Routing technique is used in LEACH Protocol, which needs high energy for data transmission from $\mathrm{CH}$ to $\mathrm{BS}$ directly in case of large network.

- Selection of $\mathrm{CH}$ in any round is random and does not consider energy level of node, which can lead to drainage of a particular node.

- Dynamic clustering technique is used in LEACH which results in extra overhead like selection of CHs and advertisement.

\section{DESCENDANTS OF LEACH PROTOCOL}

LEACH is an efficient protocol for wireless sensor networks. A lot of research work has been done to improve LEACH Protocol which have been discussed in brief as follows.

\section{A. LEACH-C Protocol}

Heinzelman et.al. [3] proposed centralized LEACH (LEACH-C) in which Cluster Head $(\mathrm{CH})$ is selected by the Base Station (BS). BS receives state, location and remaining energy information from all sensor nodes. Mean value of networks energy is calculated at the base station to select higher energy node as a $\mathrm{CH}$. Though it enhances the lifetime but requires GPS involvement.

\section{B. LEACH with deterministic cluster-head selection}

Handy et.al. [4] explored low energy adaptive clustering hierarchy with deterministic cluster-head selection to enhance lifetime of network. This protocol reduces energy consumption of wireless micro-sensor networks by using a deterministic cluster head selection algorithm. The deterministic cluster head selection 
algorithm uses reduced threshold value as follows:

$$
T(n)=\frac{p}{1-p\left(r \bmod \left(\frac{1}{p}\right)\right)} * \frac{E_{n \_ \text {current }}}{E_{n \_ \text {max }}}
$$

Where $E_{n_{-} \text {current }}$ is the current energy level and $E_{n \_ \text {max }}$ is the initial energy level of node.

C. Power Efficient Communication Protocol for Data Gathering on Mobile Sensor Network

Liu \& Lee [5] suggested Power Efficient Communication Protocols for Data Gathering on Mobile Sensor Networks. In this protocol each mobile sensor node is equipped with a GPS device to locate it in the network. Each mobile node calculates its distance from all its neighbours. In this protocol a round is categorized as invalid round, valid round and super round. A round that has not any $\mathrm{CH}$ is called as an invalid round (CM-IR) since energy is consumed without being useful. On the other hand, a valid round elects some $\mathrm{CH}$. A valid round followed by some consecutive invalid rounds in cluster head election phase is called as super round.

\section{TL-LEACH Protocol}

Loscri et.al. [6] presented Two-Level LEACH (TL$\mathrm{LEACH}$ ) protocol which uses two types of $\mathrm{CHs}$ in the network as first level $\mathrm{CHs}$ and second level $\mathrm{CHs}$. Each sensor node decides to be a primary (second level $\mathrm{CH}$ ) or secondary (first level $\mathrm{CH}$ ) or simple node (SN). First level $\mathrm{CH}$ decides which second level $\mathrm{CH}$ it will join; similarly each simple node also decides which first level $\mathrm{CH}$ it will join. Data transfers from first level $\mathrm{CH}$ to the $\mathrm{BS}$ via second level $\mathrm{CH}$. Fig. 5 shows the nodes formation after the cluster setup phase is completed.

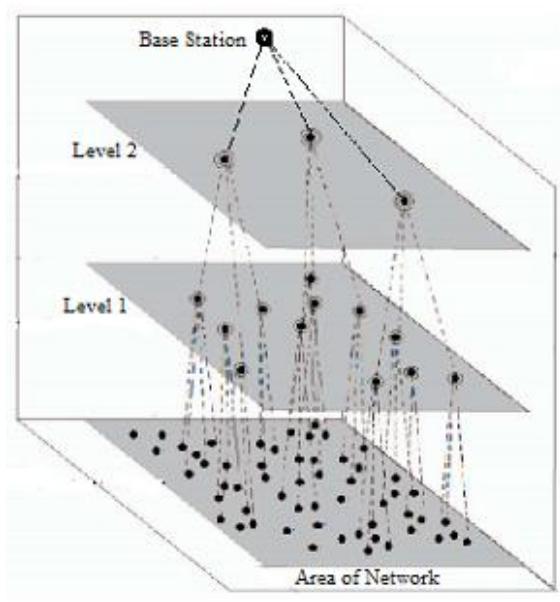

Fig.5. Architecture of TL-LEACH Protocol

\section{E. EECS Protocol}

(Ye et.al., 2005) [7] used an energy efficient clustering scheme in wireless sensor networks (EECS) to optimize network lifetime. It selects cluster heads having more residual energy through local radio communication in an automated manner without any iteration. It also provides a good distribution of cluster heads and load among cluster head is balanced by using a distance based method.

\section{F. LEACH-M Protocol}

(Kim \& Chung, 2006) [8] considered LEACH-M (Mobile LEACH) protocol with same threshold formula which was used in LEACH protocol. To avoid the availability of node during data transmission phase it uses TDMA scheduling to confirm whether a mobile node is in communication range of $\mathrm{CH}$ or not. $\mathrm{CHs}$ sends REQDATA-TRANSMITION message at starting point of each TDMA slot. If two successive TDMA frames are missed by the node then node considers itself as out of range and is removed by the member list of $\mathrm{CH}$.

\section{G. Energy-LEACH Protocol}

(Xiangning and Yulin, 2007) [9] discussed EnergyLEACH (E-LEACH) as an enhancement of LEACH by considering the residual energy of each node to select $\mathrm{CHs}$. It uses a better way to select $\mathrm{CHs}$ after first round but dissipates enough amount of energy to calculate residual energy of each node.

\section{H. MELEACH Protocol}

(Chen \& Shen, 2007) [10] discussed MELEACH Protocol which uses shortened communication distance between sensor nodes to improve load balancing. The direct communication between $\mathrm{CHs}$ and $\mathrm{BS}$ reduces capability of larger WSNs.

\section{EEPSC}

(Zahmati et.al., 2007) [11] designed an energy efficient protocol with static clustering for wireless sensor network (EEPSC). It does partitioning of entire network into few static clusters to eliminate the overhead of dynamic clustering and tries to distribute the load among by choosing high energy sensor nods as $\mathrm{CHs}$.

\section{J. MELEACH-L Protocol}

(Chen \& Shen, 2008) [12] introduced a Large-scale WSNs (MELEACH-L) protocol as an extension to MELEACH in which size of each cluster is controlled and $\mathrm{CHs}$ are separated from backbone nodes by constructing backbone tree. Channel assignment problem among neighbour clusters and the cooperation among $\mathrm{CHs}$ during data collection is also been solved in this protocol.

\section{K. LEACH-ME Protocol}

(Kumar et.al., 2008) [13] proposed LEACH-ME (Mobile Enhanced) protocol as an enhanced version of LEACH-M by selecting the less mobile nodes as $\mathrm{CHs}$ relatively to its neighbours. Each node broadcasts their IDs and estimates the distance to all other nodes. Now each node calculates mobility factor according to

$$
M_{i}(t)=\frac{1}{N-1} * \sum_{j=0}^{N-1} d_{i j}(t)
$$

Where $\boldsymbol{M}_{\boldsymbol{i}}(\boldsymbol{t})$ is the mobile factor based on remoteness of node $\mathrm{i}$ to all other nodes, $\mathrm{N}$ is the number of neighbours of node $\mathrm{i}$ and $\boldsymbol{d}_{\boldsymbol{i} \boldsymbol{j}}(\boldsymbol{t})$ is the distance of node $\mathrm{i}$ 
from its neighbours j. Nodes with least mobility factor and higher energy level are selected as $\mathrm{CHs}$.

\section{EWC Protocol}

(Cheng et.al., 2008) [14] explored Energy Efficient Weight Clustering (EWC) protocol as an extension to LEACH protocol in which residual energy, distance, and node degree are considered as metrics to select a $\mathrm{CH}$. Nodes having more neighbours or with higher degree can serve more nodes which will save more energy.

\section{EECED Protocol}

(Buyanjargal \& Kwon, 2009) [15] suggested Energy Efficient Clustering Algorithm for Event Driven (EECED). It enhances lifetime by balancing the energy usage of node. Base station is located in the centre of area and is capable of processing messages with enough memory. Data is transferred from nodes to $\mathrm{CHs}$ only when an event occurs.

\section{N. EE-RRT Protocol}

(Xian-Tian et.al., 2009) [16] presented A Novel Energy Efficient Redundant Routing Tree for WSNS (EE-RRT) which uses virtual grid with a redundant routing tree to optimize WSN lifetime. It divides each cluster into $\mathrm{N} \times \mathrm{N}$ square area (grids) and $\mathrm{CH}$ is being selected in each grid to reduce redundant information. Data transfer between $\mathrm{CH}$ and BS takes place via a transmission agent which uses a redundant routing tree to reduce dynamic clustering time.

\section{O. V-LEACH Protocol}

(Yassein et.al., 2009) [17] used V-LEACH (ViceLEACH) protocol which has an alternative cluster head Vice- $\mathrm{CH}$ along with active $\mathrm{CH}$ in each cluster. Vice- $\mathrm{CH}$ becomes $\mathrm{CH}$ to avoid isolation of cluster nodes from network in case $\mathrm{CH}$ dies. It insures the availability of cluster nodes and does not select a new $\mathrm{CH}$ each time when the $\mathrm{CH}$ dies.

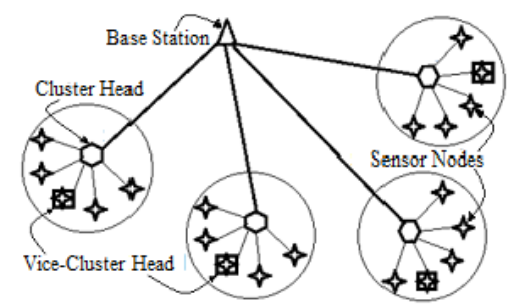

Fig.6. Architecture of V-LEACH Protocol

\section{P. NECHS Protocol}

(Hu et.al., 2009) [18] considered Energy-Efficient Cluster Head Selection (NECHS) protocol which is based on fuzzy logic. At the time of input data fuzzy logic is applied i.e. inputs are fuzzified using rules of inference and the original output can be received by applying defuzzy logic i.e. processed output is de-fuzzified to get crisp output. Residual energy and degree of nodes are taken as input to the fuzzy model. At the time of cluster formation the probability for being a $\mathrm{CH}$ is calculated by each node which is based on its remaining energy and node degree. Node having the higher probability is likely to become a $\mathrm{CH}$.

\section{Q. T-LEACH Protocol}

(Hong et.al., 2009) [19] discussed threshold based cluster head replacement for wireless sensor networks ( $\mathrm{T}$ LEACH) to enhances the network lifetime by minimizing the number of cluster head. The minimum number of $\mathrm{CHs}$ is selected by using higher threshold of residual energy. T-Leach reduces the head selection amount and replacement cost.

\section{R. DSMS Routing Protocol}

(Zhang et.al., 2009) [20] designed a distributed singlehop-multi-hop switch (DSMS) routing protocol for wireless sensor networks. In DSMS routing protocol the entire sensor network area is divide into sub area called as rings. All rings are of same radius. Each sensor nodes in the outer region or outer ring just forward the data to inner rings by using either single-hop communication or multi-hop communication. The mode of communication between a node and cluster head node is decided by the node.

\section{S. EBC Protocol}

(Nazir \& Hasbullah, 2010) [21] introduced energy balanced clustering (EBC) in wireless sensor network for cluster formation, cluster head selection inter cluster communication and intra cluster communication. $\mathrm{CHs}$ are selected based on residual energy of node, number of linked nodes or neighbours node, and distance between node and base station.

\section{T. W-LEACH Protocol}

(Abdulsalam \& Kamel, 2010) [22] proposed weighted LEACH (W-LEACH) for handling continuous, uniform and non-uniform data flow or data stream using data stream aggregation algorithm. It uses centralized approach to control non uniform network. While selecting the $\mathrm{CHs}$ nodes have their weight metrics which can be decided by residual energy level and density of nodes (number of surrounding nodes). A node with higher density will be weighted as higher node.

\section{U. N-LEACH Protocol}

(Yulan \& Chunfeng, 2010) [23] explored N-LEACH as an extension to LEACH protocol by improving cluster head election process. It considers the remaining energy of node as a parameter to choose $\mathrm{CHs}$ and maintains a minimum spanning tree which root node is $\mathrm{CH}$ of cluster.

\section{LEACH-B Protocol}

(Tong \& Tang, 2010) [24] suggested LEACH-B (Balanced-LEACH) protocol which enhances LEACH by finding the optimal number of $\mathrm{CHs}$ which can be selected by using residual energy of nodes. Minimum and optimal number of $\mathrm{CHs}$ are selected from the list of candidate $\mathrm{CH}$ nodes which are arranged in decreasing order of residual 
energy. By using optimal number of clusters LEACH-B ensures cluster balancing which saves energy consumption.

\section{W. LEACH-P Protocol}

(Cai \& Zhu, 2010) [25] presented LEACH-P (Performance) protocol with a different probability function i.e. probability function used in Energy Aware Multipath Routing (EAMR) [26] to select $\mathrm{CH}$. This new probability function optimizes the choice of selecting $\mathrm{CHs}$ and cluster rebuilding. The best energy aware path is chosen by EAMR protocol between source node and destination node. EAMR uses two energy saving mechanisms as sleep control mechanism and data transmission control mechanism. The optimal energy saving can be achieved only through the cooperation between these two mechanisms. Each node calculates the selection probability for next hop node in the routing table according to

$$
P_{N_{j}, N_{i}}=\frac{1 / C_{N_{j}, N_{i}}}{\sum_{k \epsilon F T_{j}} C_{N_{j}, N_{i}}}
$$

Where node $\boldsymbol{N}_{\boldsymbol{j}}$ select node $\boldsymbol{N}_{\boldsymbol{i}}$ as the probability of the next hop. $C_{N_{j}, N_{i}}$ represents the cost of sending data to the destination node by node $N_{j}$ though node $N_{i}$ and FT is optional nodes in the routing table.

The threshold value is calculated with the selection probability as:

$$
T(n)=\left\{\begin{array}{c}
\frac{p}{1-p\left(r \bmod \left(\frac{1}{p}\right)\right)} * \frac{1 / C_{N_{j}, N_{i}}}{\sum k \epsilon F T_{j} C_{N_{j}, N_{i}}} \\
\text { if } n \in G
\end{array}\right.
$$

X. An Adaptive Cluster Based Routing Scheme for Mobile Wireless Sensor Networks

(Kumar et.al., 2010) [27] used an adaptive cluster based routing scheme for mobile wireless sensor networks which considers the relative direction of the node mobility to improve the cluster formation. Each node broadcasts there IDs twice to estimate its distance to neighbours by using RSSI (Received Signal Strength Indication). Each node calculates relative distances from both received signals. A negative value shows that the two nodes are moving away from each other and a positive value shows that both nodes are moving towards each other and a zero value shows that they are stationary. A node declare itself as a $\mathrm{CH}$ only if there are more number of nodes which are stationary or moving towards a node. Each node decides its relative direction to its neighbours based on mobility factor $\mathrm{M}_{\mathrm{i}}(\mathrm{t})$ which is calculated as:

$$
M_{i}(t)=1-\frac{\text { No.of nodes moving away from } i}{N}
$$

Where $\mathrm{N}$ is the number of neighbours for node $\mathrm{i}$.

If $M_{i}(t)>0.5$, then there are more number of nodes which are stationary or moving towards node i. Node i declare itself as a $\mathrm{CH}$ and broadcasts $\mathrm{CH}-\mathrm{ADV}$ message. After receiving this advertisement message all nodes calculate a value which helps it to decide the $\mathrm{CH}$ using following formula:

$$
\frac{d_{i} C H(t 1)-d_{i} C H(t 2)}{t 2-t 1}
$$

Based on this value, each node decides to join a $\mathrm{CH}$ by transmitting JOIN-REQ message.

\section{Y. MR-LEACH Protocol}

(Farooq et.al., 2010) [28] considered MR-LEACH (Multi-hop Routing with LEACH) which uses multi-hop data transmission technique to send data from $\mathrm{CH}$ to $\mathrm{BS}$. At the beginning of a round and before cluster formation each node builds a table which holds the information like node's ID, residual energy level, and node's status. The table of each $\mathrm{CH}$ holds the nodes ID's of its member nodes. Status of a node can be one of three values: unknown, cluster member or $\mathrm{CH}$. $\mathrm{CH}$ is selected by using residual energy of node. $\mathrm{CHs}$ which are near to $\mathrm{BS}$ are layer-one $\mathrm{CHs}$ and which are away from BS are layer-two CHs.

\section{Z. $H A B R P$}

(Ben Alla et.al., 2011) [29] discussed a hierarchical adaptive balanced energy efficient routing protocol (HABRP) for heterogeneous wireless sensor network which uses some high energy nodes called as NCG nodes (Normal/Cluster/Gateway Node) as gateway between cluster head node and base station. NSG gateways receive aggregated data from $\mathrm{CHs}$ and forward the collected data to the base station.

\section{AA. An algorithm for lifetime optimization of WSN}

(Raju et.al., 2011) [30] designed an algorithm for lifetime optimization of wireless sensor network by considering three optimization metrics routing tree, data gathering and trade-off. Routing tree is maintained by using residual energy and distance. Data gathering is done in an energy efficient manner. The trade-off between energy consumption and data quality has been taken into consideration to optimize the lifetime of wireless sensor network.

\section{AB. LEACH-MF Protocol}

(Yan \& Liu, 2011) [31] introduced LEACH-MF as an enhancement of LEACH protocol by balancing energy consumption for large scale Wireless Sensor Network. It uses multi-layer clustering to eliminate the data redundancy at intermediate cluster heads during multihop communication.

\section{AC. I-LEACH Protocol}

(Kumar \& Kaur, 2011) [32] proposed an improvedLEACH (I-LEACH) protocol which uses residual energy of node to select $\mathrm{CH}$ node. It also considers the coordinate of node to select the nearest $\mathrm{CH}$ which requires GPS involvement to locate node. 
AD. An energy efficient hybrid MAV protocol for WSN containing mobile nodes

(Srikanth et.al., 2011) [33] explored an energy efficient hybrid MAC protocol for WSN containing mobile nodes. It uses both TDMA and CSMA/CA protocol for maximum utilization of channel to enhance the network lifetime. It utilizes the slot allotted by adjusting the frame length which is based on mobility of node and has capability of ignorance of that node which can leave the cluster.

\section{AE. EEEPSC Protocol}

(Chaurasiya et.al. 2011) [34] suggested an enhanced energy efficient protocol with static clustering for WSN (EEEPSC) as an extension to EEPSC Protocol. It does partitioning of network into distance based static clusters. It considers the spatial distribution of sensors and residual energy while selecting the $\mathrm{CHs}$ to fix it in the centre of cluster. The main objective of this protocol is to reduce the intra cluster communication overhead.

\section{AF. Far-Zone LEACH Protocol}

(Katiyar et.al., 2011) [35] presented far-zone LEACH (FZ-LEACH) as an enhancement of LEACH protocol to make it for large area network. This protocol creates a far zone of sensor nodes placed away from BS and have lesser energy than the threshold energy level to transfer the data. This threshold value is decided by the average of the minimum reachability power of every node.

\section{AG. A modified LEACH protocol using Chaos-PSO}

(Liu et.al., 2011) [36] used a modified LEACH by using Chaos-PSO (Particle swarm optimization) algorithm [37] as cluster head selection mechanism. It considers few parameters like residual energy of nodes, distance of node from base station or sink node and the maximum range of the cluster. The cluster size is decided by the signal strength.

\section{AH. Zone Division Multi Hop Hierarchical Clustering for Load Balancing}

(Ghosh et.al., 2011) [38] considered an Energy Efficient Zone Division Multi hop Hierarchical Clustering Algorithm for Load Balancing which divides the network into four zones with their centre. These four zones can or cannot be divided again by the protocols based on the efficiency of $\mathrm{CH}$.

\section{AI. ECHSSDA Protocol}

(Maraiya et.al., 2011) [39] discussed an Efficient Cluster Head Selection Scheme for Data Aggregation in Wireless Sensor Networks (ECHSSDA) using reclustering technique to reduces the overhead of clustering process, load over $\mathrm{CH}$ and energy consumption within cluster. It has an associated $\mathrm{CH}$ will become a $\mathrm{CH}$ in next round if the energy level of original $\mathrm{CH}$ becomes lower than the average energy which is computed by the BS.

\section{AJ. LEACH-GA Protocol}

(Liu \& Ravishankar, 2011) [40] designed LEACH-GA protocol using a genetic algorithm-based clustering technique to improve energy efficient protocols. Each node sends its node ID, location information and $\mathrm{CH}$ decision based on optimal percentage $\mathrm{CH}$ to the BS. BS applies genetic algorithm operations on received information to find out the optimal threshold probability and broadcast it for cluster formation.

\section{AK. LEACH-SM Protocol}

(Bakr \& Lilien, 2011) [41] introduced LEACH-SM protocol as an extension to LEACH by considering an efficient management of spares. It adds an extra phase to LEACH called as the spare selection phase that follows the setup phase and is followed by the steady state phase. The decentralized energy-efficient spare selection technique is used to select spare parts which run in simultaneously on all nodes and in all clusters to make a decision about spare which maintains the above-threshold target coverage. All spares go asleep if not in use to conserve energy.

\section{AL. ER-LEACH Protocol}

(Al-Refai et.al., 2011) [42] proposed Efficient-Routing Leach (ER-LEACH) protocol which enhances $\mathrm{CH}$ selection process by reducing overhead with load balancing. Cluster head selection is enhanced by taking residual energy. Overhead of dynamic clustering is reduced by using alternative $\mathrm{CH}$ which will take the role of $\mathrm{CH}$ in case the underlying $\mathrm{CH}$ dies. Load is balanced by using zone routing protocol which to discover the optimal route to the BS.

\section{AM. Cell-LEACH Protocol}

(Yektaparast et.al., 2012) [43] explored cell-LEACH protocol. In cell-LEACH protocol a cluster is divided into seven sections which are called as cells. These seven clusters are divided using one central cell of hexagonal shape and other six cells at each face of central hexagonal shape with their own cell heads which can communicate to each other directly. Cluster head is chosen among all nodes of seven cells of a single cluster and cell heads forwards the aggregated cell data to $\mathrm{CH}$. These clustering and celling is static and hence remain there for network lifetime. The only thing which changes is cell heads and cluster heads.

\section{AN. Wise-LEACH Protocol}

(Yueyun et.al., 2012) [44] suggested a Wise LEACH protocol (WLEACH) to enhances LEACH protocol by considering residual energy level of node at the time of $\mathrm{CH}$ selection. It also adds the multi jump routing between nodes and sleep wakeup awareness or dormancy of cluster head node.

\section{AO. Improved Far-Zone LEACH Protocol}

(Yoo et.al., 2012) [45] presented an Improved Far Zone LEACH as an extension to FZ-LEACH by using quadrant in far zone in the network. The far zone is 
created by considering the same parameter of average minimum reachability power. Far zone is further divided into quadrants. Far zone Cluster head node will be selected in the highly dense quadrant with highest power in that quadrant.

\section{AP. Improvement of LEACH protocol based on uneven clustering algorithm}

(Zhou et.al., 2012) [46] used an improvement of LEACH protocol based on uneven clustering algorithm which considers the distance between node and base station which helps in formation of clusters of different sizes. Those clusters which are nearby the base station will be small in size than the clusters which are far away from base station. By reducing the nearby cluster size energy depletion of cluster head node in case of multihop communication can be reduced which will prolong the network lifetime.

\section{AQ. Energy efficient cluster based routing protocol}

(Zhao et.al., 2012) [47] considered an energy efficient cluster based routing protocol by improving the cluster set up phase and data transmission phase. A timer is introduced to select the optimal sensor node as a cluster node at the cluster setup phase. Timer will be fixed such that if one node is listening then other node will be in sleep mode. In data transmission phase both single-hop communication and multi-hop communication technique will be used to optimize the network lifetime.

\section{AR. Approach for improvement in LEACH protocol for WSN}

(Munjal \& Malik, 2012) [48] discussed an approach for improvement in $\mathrm{LEACH}$ protocol in which $\mathrm{CH}$ selection is based on node's residual energy and it's distance from the base station. Each node sends it's residual energy level with a time stamp and expected lifetime to BS which calculates difference between current time stamp and received time stamp. If it is greater than remaining lifetime then node becomes non $\mathrm{CH}$. Hence, a better $\mathrm{CHs}$ can be selected which will prolong the network lifetime.

\section{AS. Enhanced-LEACH Protocol}

(Pawar \& Kasliwal, 2012) [49] designed an EnhancedLEACH Protocol (En-LEACH) by using modified cluster head selection phase and modified data transmission phase. It considers the energy parameter at the time of cluster setup phase. The probability function of becoming a cluster head is modified as ratio of node's energy level and aggregate energy of the cluster in the network. At the time of data transmission from cluster head. Each cluster head checks its energy level before sending data to base station. If energy level of cluster head is less than the threshold value then cluster head does not forward the data to base station and waits for the next round. By waiting for next round it avoids the probability of failure of cluster head.

\section{AT. ICCA Protocol}

(Jian-Zhen et.al., 2012) [50] introduced a new protocol called as improvement of cluster heads choosing algorithm based on LEACH protocol (ICCA) by using a better cluster head selection approach which can distribute the energy node evenly among all the nodes. It reduces the processing time by maintaining a remaining energy table and enhances the network lifetime. Only drawback of this protocol is that it takes extra space to store the remaining energy table and extra time to update this table.

\section{$A U$. Improved-LEACH}

(Gajjar et.al., 2012) [51] proposed Improved-LEACH protocol which enhances the LEACH protocol by considering the remaining energy level of node during $\mathrm{CH}$ process with it's distance from the base station. The steady state phase starts only if the sensed value of a node is greater the threshold value fixed or set by the user at the application layer. The network area is divided into four quadrants and base station is considered in one quadrant. All CHs of other quadrant uses two hop communication but $\mathrm{CH}$ of those quadrant in which $\mathrm{BS}$ is located will communicate directly to $\mathrm{BS}$.

\section{AV. EL-LEACH Protocol}

(Quynh et.al., 2012) [52] explored energy and load balance LEACH (EL-LEACH) protocol with a modified $\mathrm{CH}$ selection function in which it considers the residual energy of node ad distance between two $\mathrm{CHs}$ to avoid data redundancy. It uses an immediate cluster selection scheme if two cluster heads are close to each other in which a non $\mathrm{CH}$ node situated away from other $\mathrm{CH}$ node with highest energy level and will become a $\mathrm{CH}$ node.

\section{AW. Multi-hop LEACH Protocol}

(Wang et.al., 2012) [53] suggested Multi-hop LEACH protocol to solve quick energy drainage problem of $\mathrm{CH}$ in single hop communication in case of large-area network. Intra-cluster communication takes place between $\mathrm{CH}$ and its cluster member nodes where $\mathrm{CH}$ receives data from all member nodes at a single-hop distance and aggregates and transmits the data directly to the BS or through intermediate CHs. Multi-hop inter-cluster communication takes place via when the distance between the $\mathrm{CH}$ and the $\mathrm{BS}$ is large and the $\mathrm{CH}$ uses intermediate $\mathrm{CHs}$ for sending data to the BS.

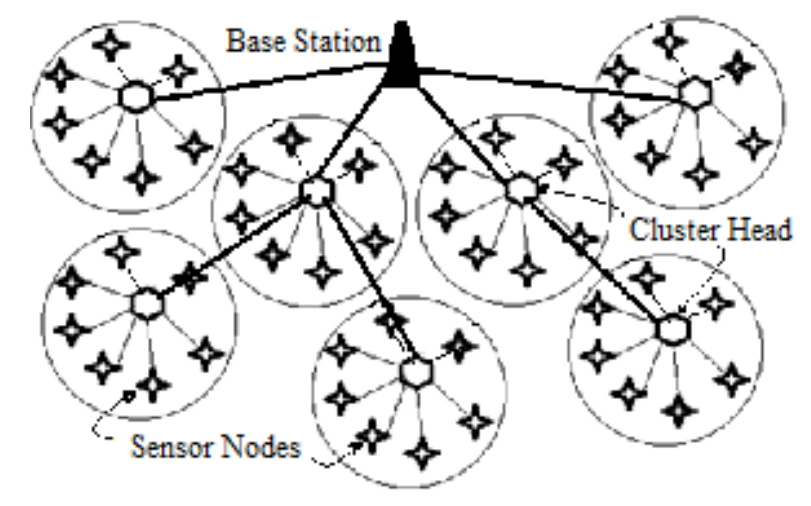

Fig.7. Architecture of Multi-hop LEACH Protocol 


\section{AX. FL-LEACH Protocol}

(Al-Ma'aqbeh et.al., 2012) [54] presented a Fuzzy Logic LEACH (FL-LEACH) as an extension to LEACH protocol which uses Fuzzy-Logic to find out the optimal number of CHs. Mamdani's fuzzy inference method [55] is used in fuzzification to determine the fuzzy sets or membership values by taking the number of nodes and network density as crisp values of the input variables. The fuzzified inputs are applied to the antecedent and consequent parts of each fuzzy rule for rule evaluation. Then aggregation is done at the output fuzzy sets using the output variable. Finally, Defuzzification of output is done at last step to get the crisp value of the output. CHs Fuzzy Logic percentage $\left(P_{F L}\right)$ can be calculated as

$$
P_{F L}=\frac{\int x * \mu_{F L} * d x}{\int \mu_{F L} * d x}
$$

Where $\mu_{F L}$ represents the aggregated output membership and $\mathrm{x}$ represents the universe of discourse.

\section{AY. LEACH-R Protocol}

(Wang \& Zhu, 2012) [56] used LEACH-R (RelayLEACH) protocol which improves the selection of $\mathrm{CHs}$ by using the selected relay nodes. A relay node is not inserted like in other protocols. A relay node is chosen by using the $\mathrm{CH}$ based on residual energy of nodes and the distance of node from BS. Selected relay node will be used for communication between the $\mathrm{CHs}$ and $\mathrm{BS}$.

\section{AZ. GCEDA Protocol}

(Mantri et.al., 2013) [57] considered Grouping of Clusters for Efficient Data Aggregation in wireless sensor network (GCEDA Protocol) to eliminates the data redundancy by using group based data aggregation method. Grouping of nodes is based on available data and correlation between cluster heads of those groups. It also uses additive and divisible data aggregation technique at each cluster head to reduce the data redundancy.

\section{BA. Q-LEACH Protocol}

(Gnanambigai et.al., 2013) [58] discussed an energy efficient algorithm based on quadrant based directional routing protocol called as Q-LEACH which divides the whole network into quadrants. Those nodes which are nearby the sink node will broadcast the message. Since it uses a reactive routing mechanism and hence all nodes maintain the destination node information before finding the path to destination or sink node. It contains advantage of both location and hierarchical based routing protocols. This protocol uses route request packet to find the path to destination.

\section{BB. iLEACH Protocol}

(Cristian \& Soni, 2013) [59] designed an improved LEACH (iLEACH) Protocol which considers the remaining energy of node to calculate the new threshold value for $\mathrm{CH}$ selection.A modified energy function is used to calculate the probability of $\mathrm{CH}$ which is based on number of rounds.

\section{BC. MODLEACH Protocol}

(Mahmood et.al., 2013) [60] introduced a modified LEACH (MODLEACH) which enhances LEACH protocol by introducing efficient $\mathrm{CH}$ replacement scheme and dual transmitting power level. Cluster head selection is based on a threshold value. Using this threshold value a node decides to remain as cluster head in next round for the same cluster if it's remaining energy is more than the threshold value. A dual transmitting power is used to transmit the amplified signal based on single hop data transmission and multi hop data transmission. Using the dual transmitting power packet drop ratio, collision rate or interference of the signals is also reduced. Later MODLEACHST and MODLEACHHT are proposed with two types of threshold values i.e. soft threshold value and hard threshold value. MODLEACHST uses soft threshold values as a pre described level of threshold and MODLEACHHT uses hard threshold value which can be greater than the defined threshold level. Both MODLEACHST and MODLEACHHT are re-active in nature or both uses event driven because transmission takes pace according to the level of threshold.

\section{BD. EBRP Protocol}

(Babaee et.al., 2013) [61] proposed a best path cluster based routing (EBPR) Protocol which uses the best energy efficient path instead of shortest path for data transmission. Minimum failure rate decides the best path between source and destination node which enhances the energy efficiency in case of high traffic.

\section{BE. DAO-LEACH Protocol}

(Saminathan et.al., 2013) [62] explored a modified LEACH protocol called as Data Aggregation and Optimal Clustering in LEACH (DAO-LEACH) which uses an effective data aggregation and optimal clustering technique. The residual energy is considered while cluster formation and cluster head selection process. It also selects an energy efficient route between source node and destination node for data transmission. For an efficient distribution of nodes in the area this protocol uses Gaussian distribution in two dimensions [63]for the node deployment. Gaussian distribution also supports node mobility.

\section{BF. MR-LEACH Protocol}

(Diane et.al., 2013) [64] suggested a measurement redundancy aware LEACH (MR-LEACH) which uses grouping of nodes based on the geographical location. At the time of data aggregation by $\mathrm{CH}$ node the information received by any node belonging to the same group processed without receiving the data from other nodes of that group. This is done because all nodes of a group contain similar information which will waste the energy in data aggregation and hence neglected.

\section{BG. EP-LEACH Protocol}

(Xiao et.al., 2013) [65] presented an energy potential LEACH (EP-LEACH) which enhances the LEACH protocol by using energy potential function while 
selecting the cluster head. This energy potential function is used to measure the capability of a node for energy harvesting. There is no limitation on a node to become a $\mathrm{CH}$ i.e. a node may become $\mathrm{CH}$ repeatedly only if it's potential energy is higher than other nodes potential energy.

\section{BH. Ad-LEACH Protocol}

(Iqbal et.al., 2013) [66] used Ad-LEACH (AdvancedLEACH) protocol for heterogeneous WSN routing which uses static clustering. $\mathrm{CH}$ in each cluster is selected by using Distributed Energy-Efficient Clustering (DEEC) [67]. Since, cluster size is small and static. Hence, CHs need to broadcast messages within a small area which reduces the power requirement of Ad-LEACH.

\section{BI. TLHCLP}

(Taneja \& Bhalla, 2013) [68] considered TLHCLP (Three Levels Hierarchical Clustering LEACH Protocol) as an enhanced version of LEACH in which BS is located at the centre of area with a pre-defined radius. All nodes are categorized in two types based on the radius from the BS. Nodes which are inside the radius is called inside nodes and those nodes which falls outside the radius is called as outside nodes. $\mathrm{CHs}$ which are situated outside the radius discover the nearest inside $\mathrm{CHs}$. Outsider $\mathrm{CH}$ sends data to $\mathrm{BS}$ through nearest inside $\mathrm{CH}$. Insider $\mathrm{CH}$ receives data from outsider $\mathrm{CH}$ and forwards data to $\mathrm{BS}$ after performing aggregation of received data.

\section{BJ. K-LEACH Protocol}

(Bakaraniya \& Mehta, 2013) [69] discussed K-LEACH protocol which optimizes the LEACH protocol by using K-Medoids clustering algorithm [70]. K-Medoids algorithm provides highly uniform clustering of nodes. This protocol considers Least distant from the centre of cluster is considered as a criterion for the selection of CHs. A highly uniform clustering will reduce the energy consumption efficiently.

\section{BK. DD-LEACH Protocol}

(Kodali \& Sharma, 2013) [71] designed DD-LEACH (LEACH with Distributed Diffusion) as an improvement over LEACH Protocol. It uses multi-hop routing of data from sensor nodes to Base station. In DD-LEACH Protocol data aggregation is done at multiple levels. First data aggregation is done at $\mathrm{CH}$ which collects the data from nodes. While forwarding data to $\mathrm{BS}$ all the intermediate $\mathrm{CHs}$ also performs data aggregation. In DDLEACH energy consumption is reduced by using multihop communication.

BL. An energy efficient optimization in LEACH architecture by using sleep wakeup based decentralized MAC protocol
(Banerjee and Bhattacharyya, 2013) [72] introduced an energy efficient optimization in LEACH architecture by using sleep wakeup based decentralized MAC protocol [73] and an efficient duty cycle [74] for each leaf nodes during cluster communication period. $\mathrm{CHs}$ have been chosen based on the residual energy of nodes. Decentralized MAC Protocol has been used for scheduling. All the nodes are in sleep mode by default but after a certain time or fixed interval nodes wakeup for data gathering. These nodes go to sleep mode again after forwarding the data to respective $\mathrm{CHs}$. By switching the node in sleep mode a lot of energy can be saved and lifetime of network can be improved.

\section{BM. EELP}

(Tumer \& Gunduz, 2014) [75] proposed Energy Efficient LEACH Protocol (EELP) which uses two threshold values as lower and upper threshold values. Lower threshold value is the minimum amount of sensed data and upper threshold value shows the danger level of data. If sensed data is below lower threshold value then the data will not be sent to base station. If value of data is more than upper threshold value then the data should be sent to base station directly. XOR operation is applied on received data from different $\mathrm{CHs}$ at Base station to avoid data redundancy.

\section{BN. Multi-Level LEACH Protocol}

(Kodali \& Aravapalli, 2014) [76] explored Multi-Level LEACH as extension to TL-LEACH. It includes 3LLEACH (Three Level LEACH) and 4L-LEACH (Four Level LEACH) protocols. Working of $3 \mathrm{~L}-\mathrm{LEACH}$ and 4L-LEACH is same as TL-LEACH, but only difference is number of levels increases between two different level $\mathrm{CHs}$. The lower level $\mathrm{CH}$ forwards aggregated data to the upper level CHs. Aggregation is performed at each level $\mathrm{CHs}$ and energy consumption is reduced because of multi-hop communication.

\section{BO. LPEDAP}

(Ramya \& Santhi, 2014) [77] suggested Localized Power Efficient Data Aggregation Tree Protocol (LPEDAP) which uses Minimum Spanning Tree (MST) technique [78] with the distributed nature of shortest weighted path based routing scheme to make routing algorithm energy efficient. This protocol is robust, scalable and self-organizing in nature.

All the descendants protocols which have been discussed above have been compared in table 1 on the basis of mobility, data transmission technique and location awareness of nodes.

Table 1. Comparison of Descendants of LEACH Protocol

\begin{tabular}{|c|c|c|c|c|}
\hline Sr. No. & Protocol Name & Mobility & $\begin{array}{c}\text { Data } \\
\text { Transmission }\end{array}$ & $\begin{array}{c}\text { Location } \\
\text { Awareness }\end{array}$ \\
\hline 1. & LEACH & $X$ & Single Hop & $\mathrm{X}$ \\
\hline 2. & LEACH-C & $\mathrm{X}$ & Single Hop & $\checkmark$ \\
\hline
\end{tabular}




\begin{tabular}{|c|c|c|c|c|}
\hline 3. & Low energy adaptive clustering hierarchy with deterministic cluster-head selection & $\mathrm{X}$ & Single Hop & $\mathrm{X}$ \\
\hline 4. & $\begin{array}{l}\text { Power Efficient Communication Protocol for Data Gathering on Mobile Sensor } \\
\text { Network }\end{array}$ & $\checkmark$ & Single Hop & $\checkmark$ \\
\hline 5. & TL-LEACH & $\mathrm{X}$ & Multi Hop & $\mathrm{X}$ \\
\hline 6. & EECS & $\mathrm{X}$ & Single Hop & $\mathrm{X}$ \\
\hline 7. & LEACH-M & $\checkmark$ & Single Hop & $\checkmark$ \\
\hline 8. & E-LEACH & $\mathrm{X}$ & Single Hop & $\mathrm{X}$ \\
\hline 9. & MELEACH & $\mathrm{X}$ & Single Hop & $\mathrm{X}$ \\
\hline 10. & EEPSC & $\mathrm{X}$ & Single Hop & $\mathrm{X}$ \\
\hline 11. & MELEACH-L & $\mathrm{X}$ & Single Hop & $\mathrm{X}$ \\
\hline 12. & LEACH-ME & $\checkmark$ & Single Hop & $\checkmark$ \\
\hline 13. & EWC & $\mathrm{X}$ & Single Hop & $\mathrm{X}$ \\
\hline 14. & EECED & $\mathrm{X}$ & Single Hop & $\checkmark$ \\
\hline 15. & EE-RRT & $\mathrm{X}$ & Single Hop & $\checkmark$ \\
\hline 16. & V-LEACH & $\mathrm{X}$ & Single Hop & $\mathrm{X}$ \\
\hline 17. & NECHS & $\mathrm{X}$ & Single Hop & $\mathrm{X}$ \\
\hline 18. & T-LEACH & $\mathrm{X}$ & Single Hop & $\mathrm{X}$ \\
\hline 19. & DSMS & $\mathrm{X}$ & Multi Hop & $\mathrm{X}$ \\
\hline 20. & LEACH-B & $\mathrm{X}$ & Single Hop & $\mathrm{X}$ \\
\hline 21. & LEACH-P & $\mathrm{X}$ & Single Hop & $\mathrm{X}$ \\
\hline 22. & An Adaptive Cluster Based Routing Scheme for Mobile Wireless Sensor Networks & $\checkmark$ & Single Hop & $\checkmark$ \\
\hline 23. & MR-LEACH & $\mathrm{X}$ & Multi Hop & $\mathrm{X}$ \\
\hline 24. & EBC & $\mathrm{X}$ & Multi Hop & $\mathrm{X}$ \\
\hline 25. & W-LEACH & $\mathrm{X}$ & Single Hop & $\checkmark$ \\
\hline 26. & N-LEACH & $\mathrm{X}$ & Single Hop & $\checkmark$ \\
\hline 27. & Zone Division Multi Hop Hierarchical Clustering for Load Balancing & $\mathrm{X}$ & Multi Hop & $\mathrm{X}$ \\
\hline 28. & ECHSSDA & $\mathrm{X}$ & Single Hop & $\checkmark$ \\
\hline 29. & LEACH-GA & $\mathrm{X}$ & Single Hop & $\mathrm{X}$ \\
\hline 30. & LEACH-SM & $\mathrm{X}$ & Single Hop & $\checkmark$ \\
\hline 31. & ER-LEACH & $\checkmark$ & Multi Hop & $\checkmark$ \\
\hline 32. & HABRP & $\mathrm{X}$ & Multi Hop & $\mathrm{X}$ \\
\hline 33. & WSN Lifetime Optimization & $\mathrm{X}$ & Multi Hop & $\checkmark$ \\
\hline 34. & LEACH-MF & $\mathrm{X}$ & Single Hop & $\mathrm{X}$ \\
\hline 35. & i-LEACH & $\mathrm{X}$ & Single Hop & $\checkmark$ \\
\hline 36. & An energy efficient hybrid MAV protocol for WSN containing mobile nodes & $\checkmark$ & Single Hop & $\checkmark$ \\
\hline 37. & EEEPSC & $\mathrm{X}$ & Single Hop & $\checkmark$ \\
\hline 38. & FZ-LEACH & $\mathrm{X}$ & Multi Hop & $\checkmark$ \\
\hline 39. & A modified LEACH protocol using Chaos-PSO & $\mathrm{X}$ & Single Hop & $\checkmark$ \\
\hline 40. & Multi-hop-LEACH & $\mathrm{X}$ & Multi Hop & $\mathrm{X}$ \\
\hline 41. & FL-LEACH & $\mathrm{X}$ & Single Hop & $\mathrm{X}$ \\
\hline 42. & LEACH-R & $\mathrm{X}$ & Multi Hop & $\mathrm{X}$ \\
\hline 43. & Cell-LEACH & $\mathrm{X}$ & Multi Hop & $\checkmark$ \\
\hline 44. & WLEACH (Wise LEACH) & $\mathrm{X}$ & Single Hop & $\checkmark$ \\
\hline 45. & Improved FZ-LEACH & $\mathrm{X}$ & Multi Hop & $\checkmark$ \\
\hline 46. & Improvement of LEACH Protocol based on uneven clustering algorithm & $\mathrm{X}$ & Multi Hop & $\checkmark$ \\
\hline 47. & Energy efficient cluster based routing protocol & $\mathrm{X}$ & Multi Hop & $\checkmark$ \\
\hline 48. & Approach for improvement in LEACH protocol for wireless sensor network & $\mathrm{X}$ & Multi Hop & $\checkmark$ \\
\hline 49. & En-LEACH & $\mathrm{X}$ & Single Hop & $\mathrm{X}$ \\
\hline 50. & ICCA & $\mathrm{X}$ & Single Hop & $\mathrm{X}$ \\
\hline 51. & Improved-LEACH & $\mathrm{X}$ & Multi Hop & $\checkmark$ \\
\hline 52. & EL-LEACH & $\mathrm{X}$ & Single Hop & $\mathrm{X}$ \\
\hline 53. & Ad-LEACH & $\mathrm{X}$ & Single Hop & $\mathrm{X}$ \\
\hline 54. & TLHCLP & $\mathrm{X}$ & Multi Hop & $\mathrm{X}$ \\
\hline 55. & K-LEACH & $\mathrm{X}$ & Single Hop & $\checkmark$ \\
\hline 56. & DD-LEACH & $\mathrm{X}$ & Multi Hop & $\mathrm{X}$ \\
\hline 57. & Sleep Wakeup Based Decentralized MAC Protocol & $\mathrm{X}$ & Single Hop & $\mathrm{X}$ \\
\hline 58. & GCEDA & $\mathrm{X}$ & Multi Hop & $\mathrm{X}$ \\
\hline 59. & Q-LEACH & $\mathrm{X}$ & Multi Hop & $\checkmark$ \\
\hline 60. & iLEACH & $\mathrm{X}$ & Single Hop & $\mathrm{X}$ \\
\hline 61. & MODLEACH & $\mathrm{X}$ & Multi Hop & $\mathrm{X}$ \\
\hline 62. & EBPR & $\mathrm{X}$ & Multi Hop & $\mathrm{X}$ \\
\hline 63. & DAO-LEACH & $\mathrm{X}$ & Multi Hop & $\mathrm{X}$ \\
\hline 64. & MR-LEACH & $\mathrm{X}$ & Single Hop & $\checkmark$ \\
\hline 65. & EP-LEACH & $\mathrm{X}$ & Single Hop & $\mathrm{X}$ \\
\hline 66. & EELP & $\mathrm{X}$ & Single Hop & $\mathrm{X}$ \\
\hline 67. & Multi-Level LEACH & $\mathrm{X}$ & Multi Hop & $\mathrm{X}$ \\
\hline 68. & LPEDAP & $\mathrm{X}$ & Multi Hop & $\checkmark$ \\
\hline
\end{tabular}




\section{CONCLUSION}

Wireless Sensor Network comprises of few sensor nodes which have capability of sensing the environment, processing the data and communicate each other. Each sensor nodes have limited power resources for which an energy efficient routing technique is required to save energy while routing the data. LEACH is a significant protocol in wireless sensor network. A lot of descendant protocols have been derived from LEACH protocol. In this paper all descendants of LEACH Protocols have been categorized on the basis of mobility of nodes, location awareness of nodes i.e. GPS involvement and data transmission method used between cluster head nodes and base station. Derived protocols which uses single hop transmission method is limited to small network area because of huge energy consumption while transferring data form cluster head to base station. Protocols using multi-hop communication technique can be used for larger networks because of less energy consumption of cluster head sensor nodes. Since, information gathered from immobile nodes may not be relevant, hence mobility of nodes makes network monitoring efficient. Although, handling mobility is a tough task which needs a mobility management model to track and communicate with mobile nodes. GPS involvement makes it easier and efficient to manage mobile networks. Mobile wireless sensor networks can be a future research field.

\section{REFERENCES}

[1] D. Estrin, R. Govindan, J. Heidemann et al., "Next century challenges: Scalable coordination in sensor networks." pp. 263-270.

[2] J. Yick, B. Mukherjee, and D. Ghosal, "Wireless sensor network survey," Computer networks, vol. 52, no. 12, pp. 2292-2330, 2008.

[3] W. B. Heinzelman, A. P. Chandrakasan, and H. Balakrishnan, "An application-specific protocol architecture for wireless microsensor networks," Wireless Communications, IEEE Transactions on, vol. 1, no. 4, pp. 660-670, 2002.

[4] M. Handy, M. Haase, and D. Timmermann, "Low energy adaptive clustering hierarchy with deterministic clusterhead selection." pp. 368-372.

[5] C.-M. Liu, and C.-H. Lee, "Power efficient communication protocols for data gathering on mobile sensor networks." pp. 4635-4639.

[6] V. Loscri, G. Morabito, and S. Marano, "A two-levels hierarchy for low-energy adaptive clustering hierarchy (TL-LEACH)." p. 1809.

[7] M. Ye, C. Li, G. Chen et al., "EECS: an energy efficient clustering scheme in wireless sensor networks." pp. 535540 .

[8] D.-S. Kim, and Y.-J. Chung, "Self-organization routing protocol supporting mobile nodes for wireless sensor network." pp. 622-626.

[9] F. Xiangning, and S. Yulin, "Improvement on LEACH protocol of wireless sensor network." pp. 260-264.

[10] J. Chen, and H. Shen, "MELEACH an energy-efficient routing protocol for WSNs," Chinese Journal of Sensors and Actuators, vol. 9, pp. 035, 2007.

[11] A. S. Zahmati, B. Abolhassani, A. A. Beheshti Shirazi et al., "An Energy-Efficient Protocol with Static Clustering for Wireless Sensor Networks," International Journal of Electronics, Circuits \& Systems, vol. 1, no. 2, 2007.

[12] J. Chen, and H. Shen, "MELEACH-L: More energyefficient LEACH for large-scale WSNs." pp. 1-4.

[13] G. S. Kumar, V. Paul, and K. P. Jacob, "Mobility metric based leach-mobile protocol." pp. 248-253.

[14] L. Cheng, D. Qian, and W. Wu, "An energy efficient weight-clustering algorithm in wireless sensor networks." pp. 30-35.

[15] O. Buyanjargal, and Y. Kwon, "An energy efficient clustering algorithm for event-driven wireless sensor networks (EECED)." pp. 1758-1763.

[16] C. Xiao-Tian, Z. Shun-Yi, W. Pan et al., "An novel energy-efficient redundant routing tree algorithm for wireless sensor networks." pp. 1-4.

[17] M. B. Yassein, A. Al-zou'bi, Y. Khamayseh et al., "Improvement on LEACH Protocol of Wireless Sensor Network (VLEACH),” JDCTA, vol. 3, no. 2, pp. 132-136, 2009.

[18] Y. Hu, X. Shen, and Z. Kang, "Energy-efficient cluster head selection in clustering routing for wireless sensor networks." pp. 1-4.

[19] J. Hong, J. Kook, S. Lee et al., "T-LEACH: The method of threshold-based cluster head replacement for wireless sensor networks," Information Systems Frontiers, vol. 11, no. 5, pp. 513-521, 2009.

[20] J. Zhang, K. Liu, X. Yang et al., "Distributed SinglehopMultihop Switch (DSMS) Routing Protocol for Wireless Sensor Networks." pp. 1-4.

[21] B. Nazir, and H. Hasbullah, "Energy balanced clustering in wireless sensor network." pp. 569-574.

[22] H. M. Abdulsalam, and L. K. Kamel, "W-LEACH: Weighted Low Energy Adaptive Clustering Hierarchy aggregation algorithm for data streams in wireless sensor networks." pp. 1-8.

[23] Z. Yulan, and J. Chunfeng, "Research about improvement of LEACH protocol." pp. 2281-2284.

[24] M. Tong, and M. Tang, "LEACH-B: An improved LEACH protocol for wireless sensor network." pp. 1-4.

[25] D. Cai, and D. Zhu, "Research and simulation of energy efficient protocol for wireless sensor network." pp. V1600-V1-604.

[26] M. Li, L. Zhang, V. O. Li et al., "An energy-aware multipath routing protocol for mobile ad hoc networks," ACM Sigcomm Asia, vol. 5, pp. 10-12, 2005.

[27] G. Kumar, A. Sitara, and K. Jacob, "An adaptive cluster based routing scheme for mobile wireless sensor networks." pp. 1-5.

[28] M. O. Farooq, A. B. Dogar, and G. A. Shah, "MRLEACH: multi-hop routing with low energy adaptive clustering hierarchy." pp. 262-268.

[29] S. Ben Alla, A. Ezzati, A. Beni Hssane et al., "Hierarchical adaptive balanced energy efficient routing protocol (HABRP) for heterogeneous wireless sensor networks." pp. 1-6.

[30] G. Raju, D. Ghosh, T. S. Kumar et al., "Wireless sensor network lifetime optimization." pp. 244-248.

[31] J.-F. Yan, and Y.-L. Liu, "Improved LEACH routing protocol for large scale wireless sensor networks routing." pp. 3754-3757.

[32] N. Kumar, and J. Kaur, "Improved leach protocol for wireless sensor networks." pp. 1-5.

[33] B. Srikanth, M. Harish, and R. Bhattacharjee, "An energy efficient hybrid MAC protocol for WSN containing mobile nodes." pp. 1-5.

[34] S. K. Chaurasiya, T. Pal, and S. D. Bit, "An enhanced energy-efficient protocol with static clustering for WSN." 
pp. 58-63.

[35] V. Katiyar, N. Chand, G. C. Gautam et al., "Improvement in LEACH protocol for large-scale wireless sensor networks." pp. 1070-1075.

[36] Z. Liu, Z. Liu, and L. Wen, "A modified leach protocol for wireless sensor networks." pp. 766-769.

[37] R. C. Eberhart, and J. Kennedy, "A new optimizer using particle swarm theory." pp. 39-43.

[38] A. K. Ghosh, A. K. Bairagi, M. A. Kashem et al., "Energy Efficient Zone Division Multihop Hierarchical Clustering Algorithm for Load Balancing in Wireless Sensor Network," International Journal of Advanced Computer Science \& Applications, vol. 2, no. 12, 2011.

[39] K. Maraiya, K. Kant, and N. Gupta, "Efficient Cluster Head Selection Scheme for Data Aggregation in Wireless Sensor Network," International Journal of Computer Applications, vol. 23, 2011.

[40] J.-L. Liu, and C. V. Ravishankar, "LEACH-GA: Genetic algorithm-based energy-efficient adaptive clustering protocol for wireless sensor networks," International Journal of Machine Learning and Computing, vol. 1, no. 1, pp. 79-85, 2011.

[41] B. A. Bakr, and L. Lilien, "LEACH-SM: A protocol for extending wireless sensor network lifetime by management of spare nodes." pp. 375-375.

[42] H. Al-Refai, A. Al-Awneh, K. Batiha et al., "EFFICIENT ROUTING LEACH (ER-LEACH) ENHANCED ON LEACH PROTOCOL IN WIRELESS SENSOR NETWORKS," International Journal of Academic Research, vol. 3, no. 3, 2011.

[43] A. Yektaparast, F.-H. Nabavi, and A. Sarmast, "An improvement on LEACH protocol (Cell-LEACH)." pp. 992-996.

[44] C. Yueyun, Z. Yue-Long, A. Jianwei et al., "An Energysaving Routing Protocol Based on LEACH." pp. 608-611.

[45] S. Yoo, "Improve Far-Zone LEACH Protocol for Energy Conserving." pp. 1-4.

[46] H. Zhou, C. Zhang, and R. Qian, "Improvement of LEACH protocol based on uneven clustering algorithm." pp. 22-26.

[47] H. Zhao, W. Zhou, and Y. Gao, "Energy Efficient and Cluster Based Routing Protocol for WSN." pp. 107-111.

[48] R. Munjal, and B. Malik, "Approach for Improvement in LEACH Protocol for Wireless Sensor Network." pp. 517521.

[49] S. Pawar, and P. Kasliwal, "Design and evaluation of enLEACH routing protocol for wireless sensor network." pp. 489-492.

[50] X. Jian-Zhen, R. Jiu-Hong, and J. Yang, "Improvement of Cluster Heads Choosing Algorithm Based on LEACH Protocol." pp. 1038-1041.

[51] S. Gajjar, K. Dasgupta, S. Pradhan et al., "Lifetime improvement of LEACH protocol for Wireless Sensor Network." pp. 1-6.

[52] T. N. Quynh, K.-H. Phung, and H. V. Quoc, "Improvement of energy consumption and load balance for LEACH in Wireless Sensors Networks." pp. 583-588.

[53] J. Wang, X. Yang, Y. Zheng et al., "An Energy-Efficient Multi-hop Hierarchical Routing Protocol for Wireless Sensor Networks," International Journal of Future Generation Communication \& Networking, vol. 5, no. 4, 2012.

[54] F. Al-Ma'aqbeh, O. Banimelhem, E. Taqieddin et al., "Fuzzy logic based energy efficient adaptive clustering protocol." p. 21.

[55] E. H. Mamdani, and S. Assilian, "An experiment in linguistic synthesis with a fuzzy logic controller,"
International journal of man-machine studies, vol. 7, no. 1 , pp. 1-13, 1975.

[56] N. Wang, and H. Zhu, "An energy efficient algrithm based on LEACH protocol." pp. 339-342.

[57] D. Mantri, N. R. Prasad, and R. Prasad, "Grouping of clusters for efficient data aggregation (GCEDA) in wireless sensor network." pp. 132-137.

[58] J. Gnanambigai, N. Rengarajan, and K. Anbukkarasi, "QLeach: An energy efficient cluster based routing protocol for Wireless Sensor Networks." pp. 359-362.

[59] A. Christian, and H. Soni, "Lifetime prolonging in LEACH protocol for wireless sensor networks." pp. 350355.

[60] D. Mahmood, N. Javaid, S. Mahmood et al., "MODLEACH: A Variant of LEACH for WSNs." pp. 158-163.

[61] E. Babaee, S. Zareei, and R. Salleh, "Best Path ClusterBased Routing Protocol for Wireless Sensor Networks." pp. 663-667.

[62] A. G. Saminathan, S. Karthik, and S. Post, "DAOLEACH: an Approach for Energy Efficient Routing based on Data Aggregation and Optimal Clustering in WSN," Life Science Journal, vol. 10, no. 7s, 2013.

[63] N. Hagen, and E. L. Dereniak, "Gaussian profile estimation in two dimensions," Applied optics, vol. 47, no. 36, pp. 6842-6851, 2008.

[64] I. Diane, R. Kacimi, Z. Mammeri et al., "Energy optimization based on the redundancy in WSNs." pp. 1-7.

[65] M. Xiao, X. Zhang, and Y. Dong, "An effective routing protocol for energy harvesting wireless sensor networks." pp. 2080-2084.

[66] A. Iqbal, M. Akbar, N. Javaid et al., "Advanced LEACH: A Static Clustering-based Heteroneous Routing Protocol for WSNs," arXiv preprint arXiv:1306.1146, 2013.

[67] L. Qing, Q. Zhu, and M. Wang, "Design of a distributed energy-efficient clustering algorithm for heterogeneous wireless sensor networks," Computer communications, vol. 29, no. 12, pp. 2230-2237, 2006.

[68] H. Taneja, and P. Bhalla, "An Improved Version of LEACH: Three Levels Hierarchical Clustering LEACH Protocol (TLHCLP) for Homogeneous WSN," International Journal of Advanced Research in Computer and Communication Engineering, vol. 2, no. 9, pp. 36103615,2013

[69] P. Bakaraniya, and S. Mehta, "K-LEACH: An improved LEACH Protocol for Lifetime Improvement in WSN," International Journal of Engineering Trends and Technology, vol. 4, no. 5, pp. 1521 - 1526, 2013.

[70] L. Kaufman, and P. Rousseeuw, Clustering by means of medoids: North-Holland, 1987.

[71] R. K. Kodali, and N. Sarma, "Energy efficient routing protocols for WSN's." pp. 1-4.

[72] R. Banerjee, and C. K. Bhattacharyya, "Energy efficient optimization in the LEACH architecture." pp. 1-6.

[73] Q. Zhao, L. Tong, A. Swami et al., "Decentralized cognitive MAC for opportunistic spectrum access in ad hoc networks: A POMDP framework," Selected Areas in Communications, IEEE Journal on, vol. 25, no. 3, pp. 589-600, 2007.

[74] Y. Li, W. Ye, and J. Heidemann, "Energy and latency control in low duty cycle MAC protocols." pp. 676-682.

[75] A. E. Tumer, and M. Gunduz, "An improved leach protocol for indoor wireless sensor networks." pp. 432437.

[76] R. K. Kodali, and N. K. Aravapalli, "Multi-level LEACH protocol model using NS-3." pp. 375-380.

[77] P. S. K.M. Ramya, "LPEDAP: Localized Power Efficient 
Data Aggregation Tree Protocol for Wireless Sensor Networks," International Journal of Advanced Research in Computer Science \& Technology, vol. 2, no. 1, pp. 229-134, 2014

[78] R. L. Graham, and P. Hell, "On the history of the minimum spanning tree problem," Annals of the History of Computing, vol. 7, no. 1, pp. 43-57, 1985.

\section{Authors' Profiles}

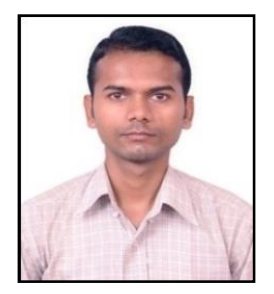

Prashant Maurya received his M.Tech degree in Computer Science and Technology from Central University of Punjab, Bathinda in 2014. His research interest includes Wireless Sensor Networks, Adhoc Networks and Network Security.

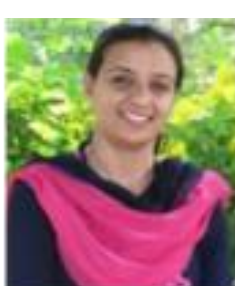

Amanpreet Kaur received her M.Tech degree in Computer Science and Engineering from Punjabi University, Patiala. She has seven year of experience as lecturer and since 2012 she is working as assistant professor at Central University of Punjab, Bathinda. Her research interest includes Adhoc Networks, Wireless Sensor Networks and Network Security.

How to cite this paper: Prashant Maurya, Amanpreet Kaur,"A Survey on Descendants of LEACH Protocol", International Journal of Information Engineering and Electronic Business(IJIEEB), Vol.8, No.2, pp.46-58, 2016. DOI: 10.5815/ijieeb.2016.02.06 